\title{
Preparation of Biomass Derived Porous Carbon: Application for Methane Energy Storage
}

\author{
Yong SUN ${ }^{a^{*}}$, Guang-Zhi SUN \\ Edith Cowan University School of Engineering, 270 Joondalup Drive Joondalup WA 6027 \\ Australia \\ ay.sun@ecu.edu.au \\ *Corresponding author
}

Keywords: Corncob, Methane storage, $\mathrm{KOH}$ activation.

\begin{abstract}
The porous carbon with high specific surface area of $3001 \mathrm{~m}^{2} / \mathrm{g}$ and pore volume of 1.59 $\mathrm{cm}^{3} / \mathrm{g}$ using corncob was prepared for methane storage. The low and high pressure adsorption equilibria were tested using the prepared carbon. The capacity of methane storage is evaluated using Tóth model with correction of fugacity using Benedict-Webb-Rubin equation of state in high pressure range. The theoretical maximum adsorption amount is achieved to be approximately 3.3 $\mathrm{mmol} / \mathrm{g}$. The adsorption process indicates physisorption dominates the adsorption with heterogeneous adsorption characteristic of sites on the surface of adsorbent at low pressure. The resultant carbon is ideal adsorbent for energy storage medium using methane.
\end{abstract}

\section{Introduction}

Natural gas is one of the most abundant natural resources in Australia [1]. The energy storage system using porous carbon with high specific surface area that regulates peak and off-peak time of gas usage is found to be a cost-effective and practical approach for energy storage system [2]. In this energy storage system, the development of highly effective adsorbents and its corresponding adsorption isotherms data especially high pressure data for methane storage are one of critical parameters for this system for the purpose of designing and sizing of adsorption column [3]. So far these data and experimental works are still limited [4]. This is our first initiation for this work. Due to abundant availabilities, neutral carbons offset and relative easiness in preparation, biomass derived porous carbon with large specific surface area has attracted many attentions in the field of energy storage [5]. Among many different precursors that shows the excellent potential large specific surface area of resultant porous carbons, corncob is one of good candidates. In our previous reports, different chemical activators have been applied to activate the precursor in order to achieve large specific surface area. Among them, $\mathrm{KOH}$ is one of the most effective chemical activator that is resulting in porous carbon with large specific surface area [6]. In this work, the preparation of resultant porous carbon by the optimal condition and evaluation of its methane storage capacity both in low and high pressure, to the best of our knowledge, have not been published before.

\section{Material and Method}

Carbon preparation. In this work, we employed our previous reported improved fast carbonization in fluidized bed followed by $\mathrm{KOH}$ chemical activation at $900{ }^{\circ} \mathrm{C}$ with carbon to $\mathrm{KOH}$ ratio of 1:1. The detailed operational procedures and parameters of fluidize bed reactor could be found in our previous reports [7]. At this optimal preparation conditions, the BET specific surface area of resultant carbon could reach $3001 \mathrm{~m}^{2} / \mathrm{g}$, with pore volume of $1.59 \mathrm{~cm}^{3} / \mathrm{g}$, and micrpore specific surface area is $2100 \mathrm{~m}^{2} / \mathrm{g}$.

Characterization of carbon. SEM morphology: Surface morphology was examined using a JSM7001F+INCA X-MAX Field emission electron microscope.

Specific surface area: The specific surface area was determined by nitrogen gas adsorption at 77 $\mathrm{K}$ at a saturation pressure of $106.65 \mathrm{kPa}$ using a Micromeritics ASAP 2020 Automated Gas Sorption 
System. The BET (Brunauer-Emmett-Teller) specific surface area was assessed within the range of relative pressures from 0.05 to 0.3 .

Gas storage. The low pressure of methane adsorption was performed by standard methods. The high-pressure adsorption measurement was performed a standard static volumetric method. Both expansion and adsorption cells have volumes of $160 \mathrm{~cm}^{3}$. The detailed procedures could be found in previous literature reports [8].

\section{Results and Discussion}

Characterization of resultant carbon. The $\mathrm{N}_{2}$ adsorption/desorption isotherm together with pore size distribution and SEM morphology of porous carbon prepared by the optimal condition is shown Fig.1.

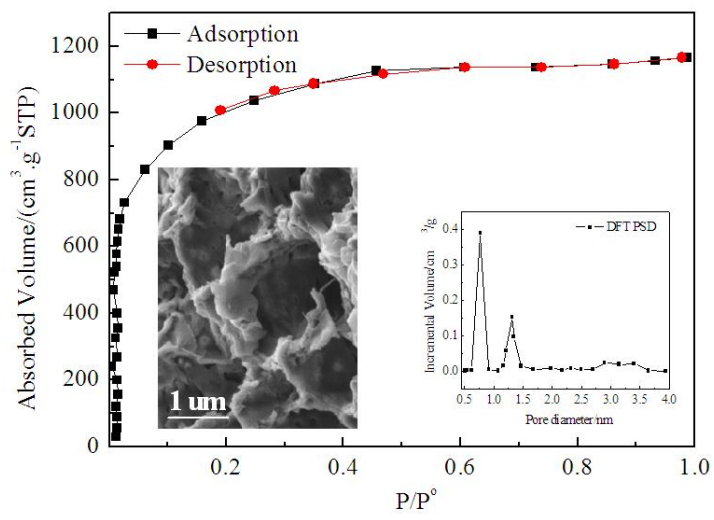

Fig.1 $\mathrm{N}_{2}$ isotherms, pore size distributions and SEM morphology of resultant carbon with the largest specific surface area.

According to Brunauer-Deming-Deming-Teller (BDDT) classification [6], the obtained isotherm presents a typical type I isotherm. The major uptake occurs at very low pressure indicating a well porous development in micro porous region. The pore size distribution by DFT (density functional theory) also indicates that the majority of pore distributes in the range of $0.2-2 \mathrm{~nm}$ range, which is the scale range of micro pore. In terms of specific surface area of carbon, BET specific surface area is $3001 \mathrm{~m}^{2} / \mathrm{g}$, the micro pore specific surface area is $2100 \mathrm{~m}^{2} / \mathrm{g}$, and corresponding external specific surface area is around $900 \mathrm{~m}^{2} / \mathrm{g}$. This result indicates that the existence of mesopores in the carbon matrix. The SEM morphology of the obtained carbon also presents a good pore formation. The channel like pore is unique for using corncob as precursor. From scale of the pores that indicated from SEM, the average is about 1um, which belongs to macro pore. The existences of these pores will facilitate infiltration during $\mathrm{KOH}$ activation, which in turns lead to a good pore development in the carbon matrix during activation.

Methane Storage and Esoteric Enthalpy Analysis. The low and high pressure adsorption equilibria of methane are measured and presented in Fig. 2a and 2b. The BWR (Benedict-WebbRubin) equation of state is adopted to determine fugacity and compressibility factor for methane in order to estimates the theoretical maximum adsorption capacity.

$$
\begin{aligned}
& p=R T \rho+\left(B_{0} R T-A_{0}-\frac{C_{0}}{T^{2}}\right) \rho^{2}+(b R T-a) \rho^{3}+a \alpha \rho^{6}+\frac{c \rho^{3}}{T^{2}}\left[\left(1+\gamma \rho^{2}\right) \exp \left(-\gamma \rho^{2}\right)\right] \\
& z=1+\left(B_{0}-\frac{A_{0}}{R T}-\frac{C_{0}}{R T^{3}}\right) \rho+\left(b-\frac{a}{R T}\right) \rho^{2}+\frac{a \alpha}{R T} \rho^{5}+\frac{c \rho^{2}}{R T^{3}}\left[\left(1+\gamma \rho^{2}\right) \exp \left(-\gamma \rho^{2}\right)\right] \\
& \ln \phi=\int_{0}^{p} \frac{(z-1)}{p} d p
\end{aligned}
$$




$$
\phi=\frac{f}{p}
$$

where $f$ is fugacity in atm, $z$ is the compressibility factor, T in $\mathrm{K}, \rho$ in mol/1, $R=0.08205$ atm $1 / \mathrm{mol}, \phi$ is fugacity coefficient, $p$ is pressure in atm. The rest of constants in Eq.1-4 are constants from BWR. By coupling the Tóth model with fugacity correlation, of which detailed derivation could be found in previous reports, the following high pressure excessive adsorption isotherm could be achieved:

$$
q=\left(\frac{q_{m}^{*} f}{\left(b+f^{n}\right)^{\frac{1}{n}}}\right)\left(q_{m}^{*}-\frac{S^{3 / 2}}{\left(q_{m}^{*} A_{v}\right)^{1 / 2}} \rho_{g}\right)
$$

Where $q$ is excessive adsorption in $\mathrm{mmol} / \mathrm{g}, b$ and $n$ are Tóth model constants, $q_{m}^{*}$ is the theoretical maximum adsorption amount in $\mathrm{mmol} / \mathrm{g}, A v$ is Avogadro number, $S$ is specific surface area in $\mathrm{m}^{2} / \mathrm{g}$.
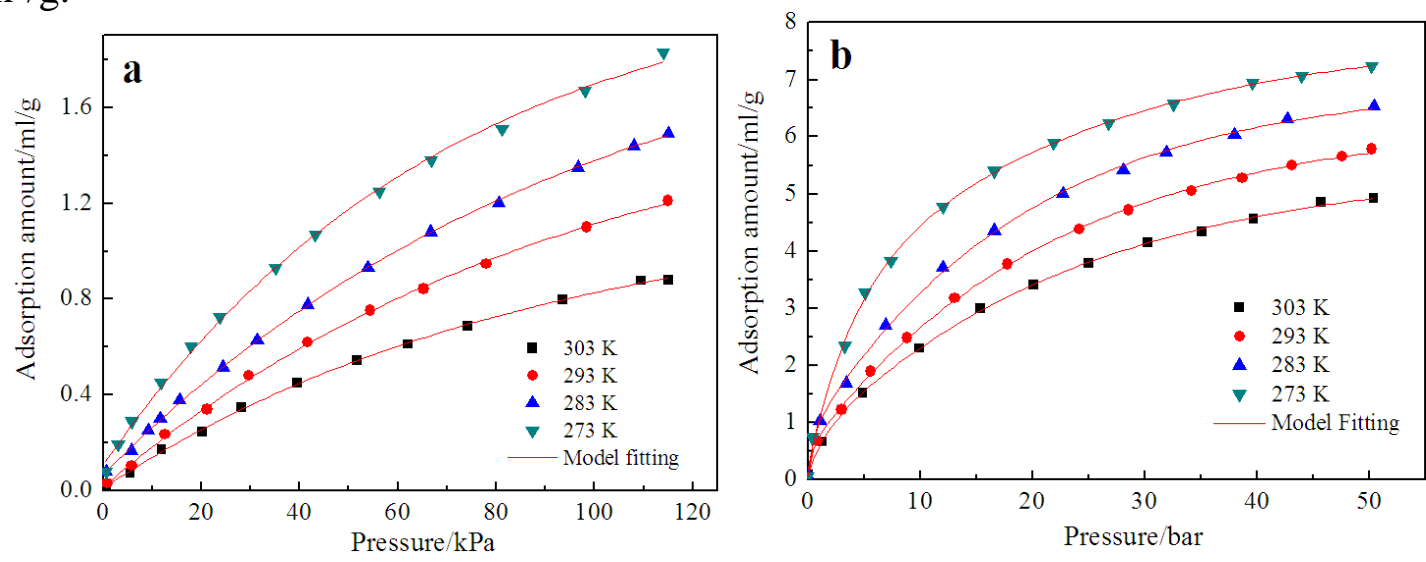

Fig.2 Tóth model fit for methane at low and high pressure.

By using generic algorithm for data regression against experimental data in Fig.2b, the theoretical maximum adsorption amount $q_{m}^{*}$ is approximately $3.3 \mathrm{mmol} / \mathrm{g}$, which is much higher than current commercial activate carbon $2.5 \mathrm{mmol} / \mathrm{g}$ at high pressure ${ }^{4}$. The enthalpy of adsorption is a significant property for characterization of the type of adsorption and degree of heterogeneity of a surface. The isosteric enthalpies of adsorption were not measured experimentally in this paper but were estimated from equilibrium data by use of the Clausius-Clapeyron equation:

$$
H_{s t}=R T^{2}\left[\frac{\partial \ln P}{\partial T}\right]_{q}
$$

By combining the isotherms with low and high pressure from Fig. 2a and Fig. 2b using Eq (6), the isosteric heat of adsorption for methane using resultant carbon with high specific surface area is shown in Fig. 3. 


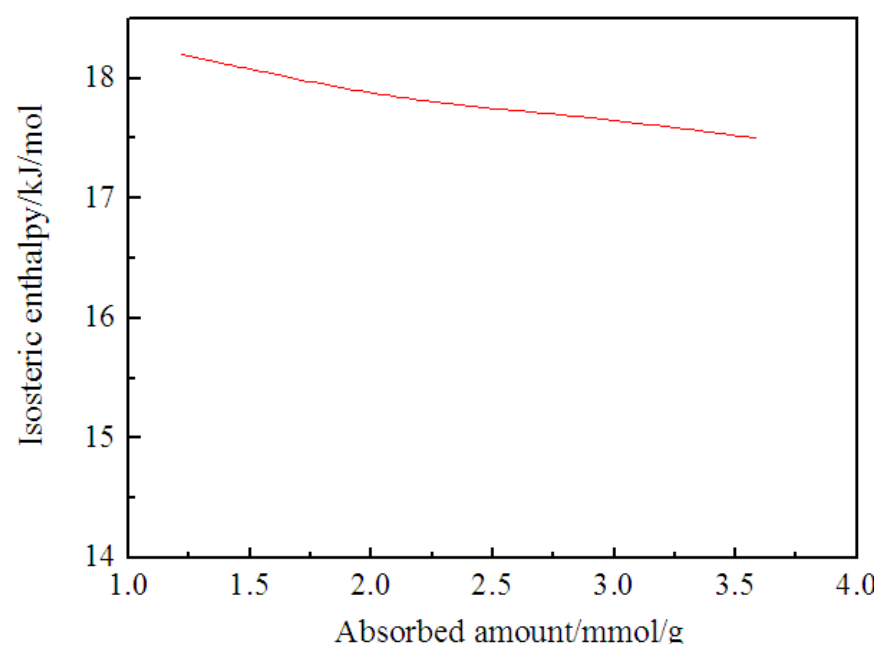

Fig.3 Isosteric enthalpies of adsorption with respect to surface loading

The result indicates that the isosteric enthalpy of adsorbing methane decreases with the increase of loading. This is a profile for heterogeneous adsorbents and is consistent with more energetic sites for methane filled preferentially at low loading and less active sites filled as adsorption proceeds, resulting in a gradual decrease in isosteric heat. From the scale of the heat of adsorption, the adsorption of methane is predominated by physisorption process which will facilitate the process of methane energy storage during peak hour desorption process for releasing methane from energy storage unit.

\section{Conclusion}

The carbon with large specific surface was prepared from corncob using $\mathrm{KOH}$ activation. The capacity of methane storage is evaluated using Tóth model with high pressure. The theoretical maximum adsorption amount $q_{m}^{*}$ is achieved to be approximately $3.3 \mathrm{mmol} / \mathrm{g}$. The adsorption process indicates physisorption process with characteristic of heterogeneous adsorption sites on the surface of adsorbent at low pressure. The result indicates that this is an ideal material and can be used as methane energy storage porous medium.

\section{References}

[1] S. Miyazaki, Coalbed methane growing rapidly as Australia gas supply diversifies, Oil. Gas. J 103, 32 (2005).

[2] H.P. Veluswamy, A.J.H. Wong, P. Babu, Rapid methane hydrate formation to develop a cost effective large scale energy storage system, Chem. Eng. J. 290161 (2016).

[3] Y. Sun, H. Chen, X. Xiao, Z.H. Li. Fractionation approaches for high value conversion of lignin: a review. J. China. Paper. and Pulp. Industry 2658 (2006).

[4] Y. Sun, P.A. Webley, Preparation of Activated Carbons with Large Specific Surface Areas from Biomass Corncob and Their Adsorption Equilibrium for Methane, Carbon Dioxide, Nitrogen, and Hydrogen. Ind. Eng. Chem. Res 509286 (2011).

[5] Y. Sun. J.P. Zhang, G. Yang, M. Li, Z.H. Li. Kinetic study on the dilute hydrochloric acid hydrolysis of corn straw xylose. Chem. Eng. 3549 (2007).

[6] Y. Sun, P.A. Webley, Preparation of activated carbons from corncob with large specific surface area by a variety of chemical activators and their application in gas storage. Chem. Eng. J 162883 (2010).

[7] Y. Sun, J.P. Zhang, G. Yang, Z.H. Li, An improved process for preparing activated carbon with large specific surface area from corncob. Chem. Biochem. Eng. Q 21169 (2007).

[8] T. Bhatelia, C. Li, Y. Sun, P. Hazewinkel, N. Burke, V. Sage, Chain length dependent olefin 
re-adsorption model for Fischer-Tropsch synthesis over Co-A12O3 catalyst. Fuel Process Technol. 125277 (2014). 